\title{
A structure-preserving energy function for stability analysis of $\mathrm{AC} / \mathrm{DC}$ systems
}

\author{
$\mathrm{K}_{\mathrm{R}}$ PADIYAR $^{1}$ and H S Y SASTRY ${ }^{2}$ \\ ${ }^{1}$ Department of Electrical Engineering, Indian Institute of Science, \\ Bangalore 560012, India \\ ${ }^{2}$ Karnataka Regional Engineering College, Srinivasanagar 574 157, India
}

\begin{abstract}
Direct stability analysis of $\mathrm{AC} / \mathrm{DC}$ power systems using a structure-preserving energy function (SPEF) is proposed in this paper. The system model considered retains the load buses thereby enabling the representation of nonlinear voltage dependent loads. The HVDC system is represented with the same degree of detail as is normaily done in transient stability simulation. The converter controllers can be represented by simplified or detailed models. Two or multi-terminal DC systems can be considered. The stability analysis is illustrated with a 3-machine system example and encouraging results have been obtained.
\end{abstract}

Keywords. Direct stability analysis; structure-preserving energy function; nonlinear voltage dependent loads.

\section{Introduction}

The application of energy functions for direct stability analysis of AC power systems has now reached a certain level of maturity (see Pai 1989, Ribbens-Pavella \& Evans 1985) where it can be used as a screening tool for both on-line and off-line studies. However, there are several issues that are yet to be fully resolved - detailed system modelling, fast computation of critical energy, accuracy of prediction and correlation of energy margins to stability limits. The present state-of-the-art application of energy functions involves prediction of either critical clearing times or energy margin for a three-phase fault at a bus followed by clearing of the fault with or without line tripping. Whereas the formulation of the energy function is relatively straightforward, the computation of critical energy is either based on (a) evaluation of unstable equilibrium point (UEP) or (b) potential energy boundary surface (PEBS) method. Both methods are strictly approximations as the trajectory of the system does not necessarily pass through the UEP. However, practical experience shows the accuracy of the results.

While most of the work on energy functions is based on the reduced model of the system assuming constant impedance loads, recent developments in defining energy functions on a structure preserving model are promising as (a) realistic load models can be considered, (b) the problem of transfer conductances is eliminated. The reader is referred to Bergen \& Hill (1981), Narasimhamurthi \& Musavi (1984), Tsolas et al 
(1985), Varaiya et al (1985), Padiyar \& Sastry (1983, 1987), Padiyar \& Ghosh (1987), Hiskens \& Hill (1989) for details. The structure preserving energy function (SPEF) has been developed for a two-axis generator model with excitation system (see Padiyar \& Ghosh 1989).

The application of energy functions for $A C / D C$ system was first attempted by Pai et al (1981) by using a simplified DC link model. The dynamic loads due to the DC link along with other loads were represented as current injections at generator internal buses of the reduced system using distribution factors. The application of SPEF with realistic DC link models was reported by Padiyar \& Sastry (1984). Ni \& Fouad (1987) have applied energy functions neglecting the DC link dynamics and assuming strong voltage support at the converter bus. DeMarco \& Canizares (1992) have recently proposed a vector energy function for $A C / D C$ systems with two components, one for $A C$ and the other for DC.

This paper presents an SPEF for AC/DC systems with two or multiterminal DC links. The energy function incorporates the DC system as dynamic loads at converter buses. Detailed or simplified converter control models can be used. Voltage dependent nonlinear loads are considered. The analysis is illustrated by an example of a 3-generator system.

\section{HVDC system model}

The HVDC system consists of two or more converters (if multiterminal operation is to be considered). A converter terminal is shown in figure 1 . In general, there is more than one bridge connected in series. All the bridges at a terminal are identical and operate at the same value of the control angle $\theta$ (delay or extinction angle).

\subsection{Converter model}

In transient stability studies, it is adequate to represent the converter by a simplified model in which the valve switchings are ignored. This is equivalent to ignoring the $A C$ and DC harmonics.

From converter theory (see Padiyar 1991) the average DC voltage (pole to ground) per unit at a converter terminal is given by

where

$$
V_{d}=k a V \cos \theta-R_{c} I_{d}
$$

$$
\begin{aligned}
k & =3 \sqrt{2} N_{s} n_{b} V_{a c b} /\left(\pi N_{p} V_{d b}\right) \\
R_{c} & = \pm\left(3 n_{b} X_{c} / \pi Z_{d b}\right), Z_{d b}=\left(V_{d b} / I_{d b}\right)
\end{aligned}
$$

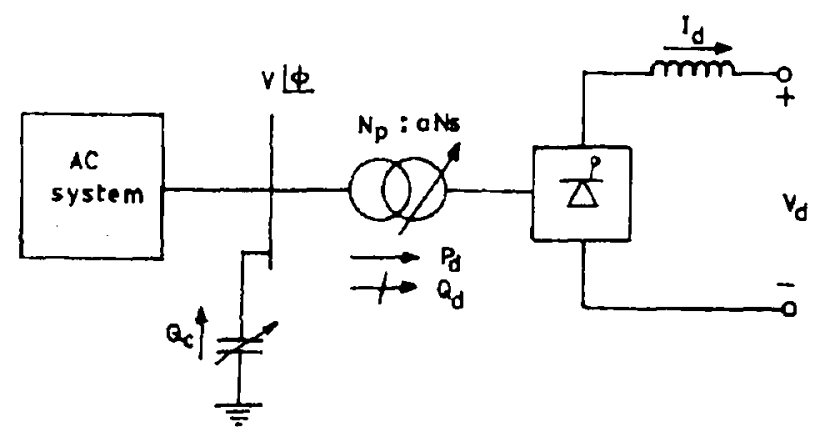

Figure 1. Single line diagram of converter station. 


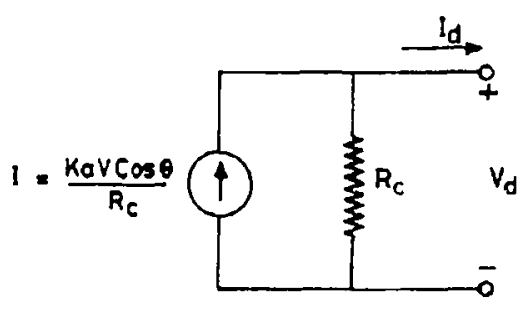

Figure 2. Norton's equivalent of a converter.

$V_{a c b}, V_{d b}, I_{d b}$ and $Z_{d b}$ are base AC voltage, DC voltage, DC current and DC impedance, respectively. $n_{b}$ is the number of bridges per terminal, $X_{c}$ is the converter transformer leakage reactance (on valve side).

For a rectifier, $\theta=\alpha$, while for an inverter, $\theta=\gamma . I_{d}$ and $R_{c}$ are assumed to be positive for the rectifier and negative for the inverter. The per unit system used here is more general than that used earlier (for example in Fudeh \& Ong 1981). This is because $V_{a c b}$ can be chosen independently of $V_{d b}$ and can assume different values for different terminals. Similarly $n_{b}, N_{s}$ and $N_{p}$ can vary depending on the terminal. The effect of these parameters is included in a single parameter $k$ which can vary. From (1), the converter terminal can be represented by Norton's equivalent of a current source $I$ in parallel with $R_{c}$ (see figure 2). The power $\left(P_{d}\right)$ and reactive power $\left(Q_{d}\right)$ are given by

$$
\begin{gathered}
P_{d}=p V_{d} I_{d}, \\
Q_{d}=P_{d} \tan \zeta, \\
\cos \zeta=V_{d} / k a V,
\end{gathered}
$$

where $p$ is the number of poles (one or two).

\subsection{DC network equations}

Unlike in AC networks, transients in a DC network are sometimes taken into account. However, it is simple to ignore fast transients and model only the resistive DC network. The use of Norton's equivalent circuits for converters, enables the DC network equations to be expressed with ground as reference node. The resistive DC network is modelled as

$$
\text { [G] } V_{d}=I \text {, }
$$

where $[G]$ is a conductance matrix of size $n_{d}$ where $n_{d}$ is the number of converter terminals. It is assumed that non-converter buses are absent or are eliminated.

\subsection{Converter control model}

There are three types of controller models.

(1) Detailed models which represent the dynamics of controllers and the parameters are tuned for particular system requirements. The use of such a model requires a knowledge of the actual system conditions for authenticity. The detailed controller model has to be interfaced with the network model considering the transients.

(2) A performance model assumes that the controllers are adequately designed to carry out the objectives of the control. For example, it is assumed that the actual DC current in the link faithfully follows the current reference instantaneously or with 


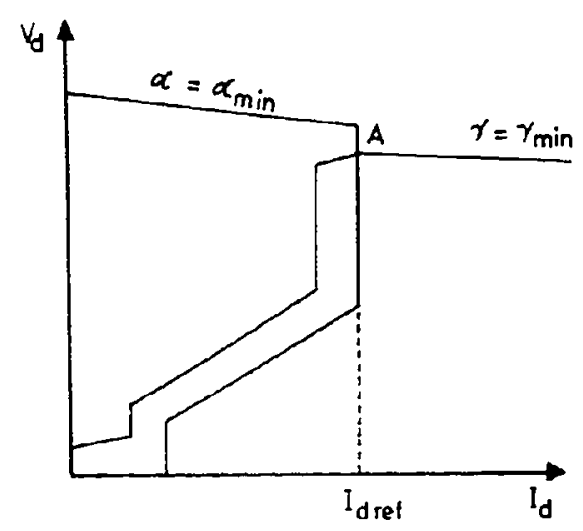

Figure 3. Control characteristics.

prespecified time delay. In the former case; it is equivalent to ignoring the controller dynamics and modelling the performance of the controller using steady-state control characteristics. Typical control characteristics in $V_{d}-I_{d}$ plane for a two-terminal system are shown in figure 3. The intersection of the rectifier and inverter characteristics defines the operating point. The operations at ' $\mathrm{A}$ ' corresponds to current control at the rectifier and constant extinction angle (CEA) control at the inverter. This is the normal operating mode. However, voltage dip at the rectifier converter bus results in the mode shift where current control is transferred to the inverter and the rectifier operates at minimum $\alpha$ (delay angle). Figure 3 also shows the influence of voltage-dependent current order limiter (VDCOL) which is mainly provided for preventing voltage instability and as a back-up protection against commutation failures.

The use of a performance model requires the use of mode shift logic to identify the transition from one mode to another. In multiterminal DC systems, the prediction of mode-shifts can be cumbersome. Hence, a variation of the performance model has been developed and is termed (Lefebvre et al 1991) a firing angle based simplified model.

(3) In the firing angle based simplified model the identity of the DC network is explicity retained. The network model can be only resistive or include inductances also.

\section{AC system model}

The following assumptions are made in modelling the AC system:

(i) the synchronous machine is represented by classical model;

(ii) the AC network is assumed to be lossless;

(iii) machine damping is neglected.

The assumption (i) is made here only for convenience and can be relaxed for detailed analysis including the effects of AVR (see Padiyar \& Ghosh 1989).

\subsection{Generator model}

The swing equation for the ith generator, using centre of inertia (COI) variables, can be written as

$$
\begin{aligned}
M_{i} \dot{\omega}_{i} & =P_{m i}-P_{e i}-\left(M_{i} / M_{T}\right) P_{\mathrm{Col}}, \\
\dot{\theta} & =\omega_{i},
\end{aligned}
$$


where

$$
\begin{aligned}
P_{e i} & =E_{i} V_{i} \sin \left(\theta_{i}-\phi_{i}\right) / x_{d i}^{\prime}, \quad i=1,2, \ldots m, \\
P_{\mathrm{COI}} & =\sum_{i=1}^{m}\left(P_{m i}-P_{e i}\right),
\end{aligned}
$$

$m$ is the number of machines.

\subsection{Load model}

The loads are modelled as arbitrary functions of the respective bus voltages. Thus,

$$
\begin{aligned}
& P_{l j}=f_{p j}\left(V_{j}\right), \\
& Q_{l j}=f_{q j}\left(V_{j}\right) .
\end{aligned}
$$

\subsection{AC network equations}

The AC network is described by the power flow equations at each bus. At converter buses, $P_{d}$ and $Q_{d}$ are treated as dynamic loads.

The power flow equations at bus $i$ are given by

$$
\begin{aligned}
& P_{i}+P_{l i}+e_{i} P_{d i}=0, \\
& Q_{i}+Q_{l i}+e_{i} Q_{d i}=0,
\end{aligned}
$$

where $e_{i}=1$, if $i \in D$ (where $D$ is the set of converter buses), and $e_{i}=0$, otherwise. $P_{i}$ and $Q_{i}$ are the injected power and reactive power given by the following expressions.

$$
\begin{aligned}
& P_{i}=m_{i} E_{i} V_{i} \sin \left(\phi_{i}-\theta_{i}\right) / x_{d i}^{\prime}+\sum_{j=1}^{N} B_{i j} V_{i} V_{j} \sin \phi_{i j}, \\
& Q_{i}=m_{i}\left(V_{i}^{2}-E_{i} V_{i} \cos \left(\theta_{i}-\phi_{i}\right)\right) / x_{d i}^{\prime}-\sum_{j=1}^{N} B_{i j} V_{i} V_{j} \cos \phi_{i j},
\end{aligned}
$$

where $m_{i}=1$, if $i \in G$ (where $G$ is the set of generator terminal buses), and $m_{i}=0$, otherwise.

\section{Structure preserving energy function}

Consider the following SPEF defined on the post-fault system.

where

$$
W(\boldsymbol{\omega}, \boldsymbol{\theta}, \mathbf{V}, \boldsymbol{\phi}, t)=W_{1}(\boldsymbol{\omega})+W_{2}(\boldsymbol{\theta}, \mathbf{V}, \boldsymbol{\phi}, t),
$$

$$
\begin{aligned}
W_{1}(\omega) & =\sum_{i=1}^{m} \frac{1}{2} M_{i} \omega_{i}^{2} \\
W_{2} & =W_{21}(\theta)+W_{22}(t)+W_{23}(\mathbf{V})+W_{24}(\boldsymbol{\theta}, \phi, v)+W_{d}(\mathbf{V}, t) \\
W_{21}(\theta) & =-\sum_{i=1}^{m} P_{m i}\left(\theta_{i}-\theta_{i 0}\right) \\
W_{22}(t) & =\sum_{i=1}^{N} \int_{t u}^{t} P_{t i}\left(\mathrm{~d} \phi_{i} / \mathrm{d} t\right) \mathrm{d} t
\end{aligned}
$$




$$
\begin{aligned}
& W_{23}(V)=\sum_{i=1}^{N} \int_{V_{i 0}}^{V_{i}}\left(f_{q i}\left(x_{i}\right) / x_{i}\right) \mathrm{d} x_{i} \\
& W_{24}(\boldsymbol{\theta}, \boldsymbol{\phi}, \mathbf{V})=\sum_{i=1}^{m}\left[\left(\left(V_{i}^{2} / 2\right)-E_{i} V_{l} \cos \left(\theta_{i}-\phi_{i}\right)\right) x_{d i}^{\prime}\right. \\
& -\left(\left(V_{i 0}^{2} / 2-E_{i} V_{i 0} \cos \left(\theta_{i 0}-\phi_{i 0}\right)\right) / x_{d i}^{\prime}\right] \\
& -\sum_{i=1}^{N} \sum_{j=1}^{N} \frac{1}{2} B_{i j}\left[V_{i} V_{j} \cos \phi_{i j}-V_{i 0} V_{j 0} \cos \phi_{i j o}\right] \text {, } \\
& W_{d}(\mathbf{V}, t)=\sum_{j \in D}\left[\int_{t_{0}}^{t} P_{d j}\left(\mathrm{~d} \phi_{j} / \mathrm{d} t\right) \mathrm{d} t+\int_{V_{j o}}^{V_{j}}\left(Q_{d j} / V_{j}\right) \mathrm{d} V_{j}\right]
\end{aligned}
$$

It can be shown that the derivative of $W$ is zero along the system trajectory. For,

$$
\begin{aligned}
& \left.\sum_{i=1}^{m}\left(\partial W_{i} / \partial \omega_{i}\right)\left(\mathrm{d} \omega_{i} / \mathrm{d} t\right)+\left(\partial W_{2} / \partial \theta_{i}\right) \cdot\left(\mathrm{d} \theta_{i} / \mathrm{d} t\right)\right) \\
& =\sum_{i=1}^{m}\left(M_{i} \omega_{i} \dot{\omega}_{i}-P_{m i} \omega_{i}+P_{e i} \omega_{i}\right)=0 \\
& \sum_{i=1}^{N}\left(\partial W_{2} / \partial \phi_{i}\right)\left(\mathrm{d} \phi_{i} / \mathrm{d} t\right)+\left(\partial W_{2} / \partial t\right)=\sum_{i=1}^{N}\left(P_{i}+P_{l i}+e_{i} P_{d i}\right)\left(\mathrm{d} \phi_{i} / \mathrm{d} t\right)=0 \\
& \sum_{i=1}^{N}\left(\partial W / \partial V_{i}\right)=\sum\left(1 / V_{i}\right)\left(Q_{l i}+Q_{i}+e_{i} Q_{d i}\right)=0
\end{aligned}
$$

\section{Comments}

(1) It is assumed that the system models are well-defined in the sense that the voltages at the load buses can be solved in a continuous manner at any given time during the transient. This means that the system trajectories are smooth and there are no jumps in the energy function.

(2) Consider the terms

$$
\int_{t_{0}}^{t} P_{l i}\left(\mathrm{~d} \phi_{i} / \mathrm{d} t\right) \mathrm{d} t \text { or } \int_{t_{0}}^{t} P_{d i}\left(\mathrm{~d} \phi_{i} / \mathrm{d} t\right) \mathrm{d} t .
$$

These can be expressed as

$$
\begin{aligned}
& \int_{t 0}^{t} P_{l i}\left(\mathrm{~d} \phi_{i} / \mathrm{d} t\right) \mathrm{d} t=P_{l i}\left(\phi_{i}-\phi_{i 0}\right)-\int_{t_{0}}^{t}\left(\mathrm{~d} P_{l i} / \mathrm{d} t\right) \phi_{i} \mathrm{~d} t \\
& \int_{t_{0}}^{t} P_{d i}\left(\mathrm{~d} \phi_{i} / \mathrm{d} t\right) \mathrm{d} t=P_{\mathrm{di}}\left(\phi_{i}-\phi_{i 0}\right)-\int_{i_{0}}^{l}\left(\mathrm{~d} P_{d i} / \mathrm{d} t\right) \phi_{i} \mathrm{~d} t .
\end{aligned}
$$

If $\left(\mathrm{d} P_{t i} / \mathrm{d} t\right)$ and $\left(\mathrm{d} P_{d i} / \mathrm{d} t\right)$ are small, the second terms on the RHS of the above equations can be neglected. This approximation has the advantage of making the SPEF pathindependent.

(3) It can be shown that

$$
W_{24}(\theta, \phi, V)=\sum_{k=1}^{n_{k}} \frac{1}{2}\left(Q_{k}-Q_{k 0}\right)
$$


where $n_{e}$ is the number of elements of the AC network including machine reactances and $Q_{k}$ is the reactive power loss in element $k$. From conservation of energy

$$
\sum_{k=1}^{n_{a}} \frac{1}{2} Q_{k}=\frac{1}{2}\left[\sum_{i=1}^{m} Q_{G l}-\sum_{j=1}^{N} Q_{i j}-\sum_{k=1}^{n_{4}} Q_{d k}\right]
$$

where $Q_{G i}$ is the reactive power generation at the internal bus of generator $i$. Equation (30) can be used to simplify the computation of SPEF.

(4) The component $W_{d}(V, t)$ of the energy function, which is attributed to the DC system, follows from treating $P_{d}$ and $Q_{d}$ at each converter bus as dynamic loads. The effect of the DC link controller is handled indirectly influencing $P_{d}$ and $Q_{d}$. Thus any controller model can be considered.

For a two-terminal DC system, neglecting DC system losses, $W_{d}$ can be expressed approximately as

where

$$
W_{d}=W_{d 1}+W_{d 2}+W_{d 3},
$$

$$
\begin{aligned}
& W_{d 1}=P_{d}\left[\left(\phi_{r}-\phi_{t}\right)-\left(\phi_{r 0}-\phi_{t 0}\right)\right], \\
& W_{d 2}=k_{r} a_{r} I_{d} \int_{V_{r 0}}^{V_{r}} \sin \zeta_{r} \mathrm{~d} V_{r}, \\
& W_{d 3}=K_{i} a_{i} I_{d} \int_{V_{i 0}}^{v_{1}} \sin \zeta_{l} \mathrm{~d} V_{l},
\end{aligned}
$$

$\phi_{l}$ and $\phi_{r}$ refer to the bus angles at rectifier and converter buses, respectively.

Assuming that the inverter controls the power factor angle $\zeta_{i}$ (by keeping it constant)

$$
\begin{aligned}
& W_{d 2}=k_{r} a_{r} I_{d} \int_{V_{10}}^{V_{r}}\left[\left(k_{r}^{2} a_{r}^{2} \cdot V_{r}^{2}-V_{d}^{2}\right)^{1 / 2} /\left(k_{r} a_{r} V_{r}\right)\right] \mathrm{d} V_{r}, \\
& W_{d 3}=k_{i} a_{i} I_{d} \sin \zeta_{i}\left(V_{i}-V_{i 0}\right) .
\end{aligned}
$$

By noting that

$$
\int\left[\left(x^{2}-a^{2}\right)^{1 / 2} / x\right] \mathrm{d} x=\left(x^{2}-a^{2}\right)^{1 / 2}-a \sec ^{-1}(x / a),
$$

the integral in (35) can be expressed as

$$
W_{\mathrm{d} 2}=k_{r} a_{r} I_{d}\left[V_{r} \sin \zeta_{r}-V_{r 0} \sin \zeta_{r 0}-\left(V_{r} \zeta_{r} \cos \zeta_{r}-V_{r 0} \zeta_{r 0} \cos \zeta_{r 0}\right)\right]
$$

It is to be noted that during a transient, the DC current $I_{d}$ is regulated by the rectifier and is assumed to be independent of $V_{r}$ and $V_{i} . V_{d}$ is dependent on $V_{i}$.

If there is a mode shift resulting in current control at the inverter and power factor control at the rectifier, the subscripts ' $r$ ' and ' $i$ ' are to be interchanged in (36) and (37).

If $\gamma$ (extinction angle) is to be maintained constant at the inverter, then $W_{d 3}$ can be expressed as

$$
\begin{aligned}
W_{d 3} & =I_{d} \int_{V_{l 0}}^{V_{1}}\left[\left(A+B V_{i}+C V_{l}^{2}\right)^{1 / 2} / V_{i}\right] \mathrm{d} V_{l}, \\
A & =-R_{c i}^{2} I_{d}^{2}, \\
B & =2 k_{i} a_{i} R_{c i} I_{d} \cos \gamma_{l}, \\
C & =\left(1-\cos \gamma_{i}^{2}\right) k_{l}^{2} a_{i}^{2} .
\end{aligned}
$$

An explicit expression for the integral of (38) can be obtained. 


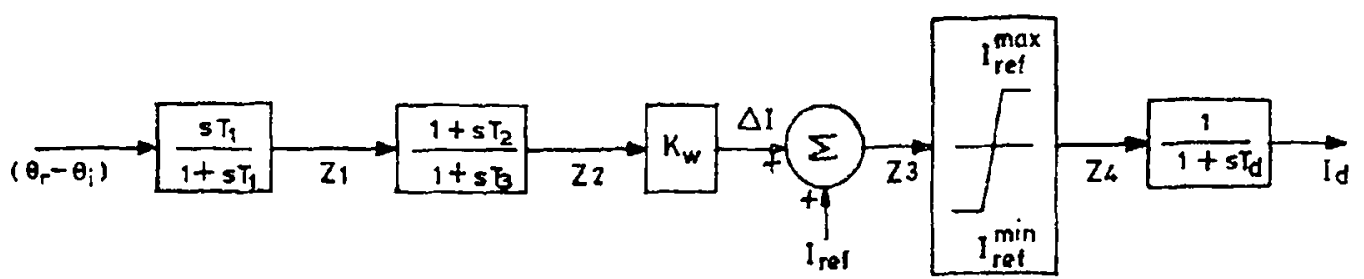

Figure 4. Block diagram of auxiliary controllcr.

\section{A numerical example}

For illustration, a 3-machine system example is adapted from Anderson \& Fouad (1977). The single line diagram of the system is shown in figure 6. DC link is connected between buses 6 and 9 . The controller model is assumed to be performance model based on DC current and current modulation using an auxiliary controller (see figure 4) or emergency controller (see figure 5) is considered. The control signal for the auxiliary controller is taken from the difference in the phase angles of the two converter buses. A washout circuit is included to eliminate steady-state offset and filter out very low frequency components that occur normally. The equations of the auxiliary controller and the emergency controller are given next.

\subsection{Auxiliary controller}

The block diagram of this controller is shown in figure 4 .

The dynamic and algebraic equations for this controller are given below.

$$
\begin{aligned}
\dot{I}_{d} & =\left(Z_{4}-I_{d}\right) / T_{d}, \\
\dot{y}_{1} & =\left[\left(\phi_{\mathrm{r}}-\phi_{i}\right)-y_{1}\right] / T_{1}, \\
\dot{y}_{2} & =\left[\left(1-T_{2} / T_{3}\right) Z_{1}-y_{2}\right] / T_{3}, \\
Z_{1} & =\left(\theta_{r}-\theta_{t}\right)-y_{1}, \\
Z_{2} & =Z_{1}+y_{2}, \\
\Delta I & =K_{w} Z_{2}, \\
Z_{3} & =I_{\mathrm{ref}}+\Delta I, \\
Z_{4} & =Z_{3}, \text { if } I_{\mathrm{ref}}^{\min } \leqslant Z_{3} \leqslant I_{\mathrm{ref}}^{\max }, \\
& =I_{\mathrm{ref}}^{\max }, \text { if } Z_{3}>I_{\mathrm{ref}}^{\max }, \\
& =I_{\mathrm{ref}}^{\min }, \text { if } Z_{3}<I_{\mathrm{ref}}^{\min } .
\end{aligned}
$$

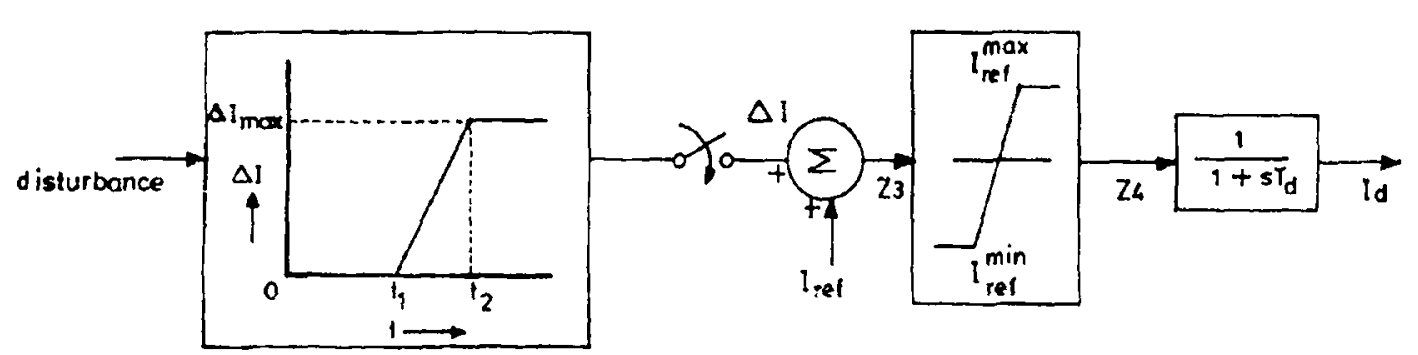

Figure 5. Block diagram of emergency controller. 


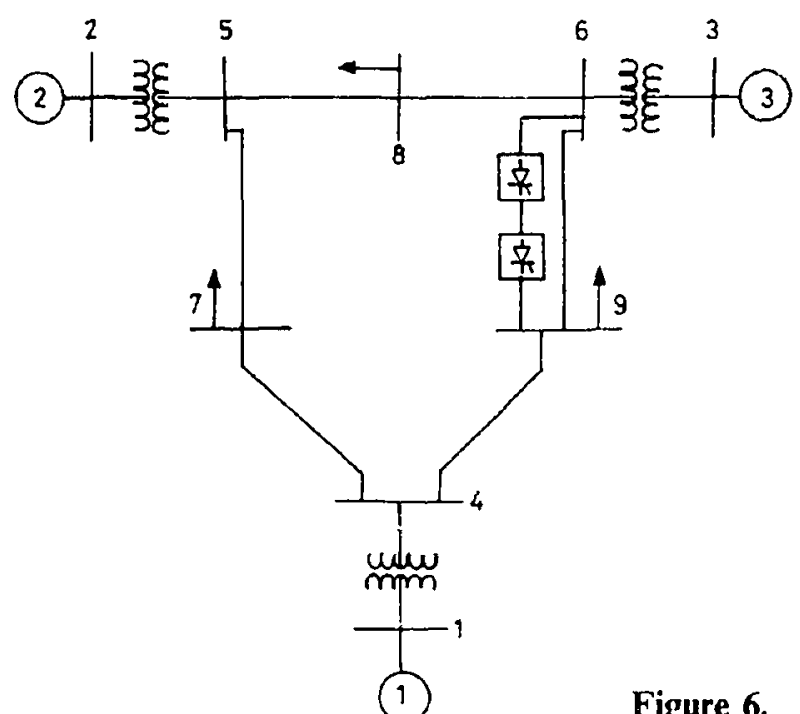

Figure 6. Single-line diagram of 3-machine system.

\subsection{Emergency controller}

This is shown in figure 5 . Here the change in the current reference $\Delta I$ increases from zero to $\Delta I_{\max }$ linearly with time, when a disturbance in the AC system is sensed.

The dynamic and algebraic cquations are given below.

$$
\begin{aligned}
\dot{I}_{d} & =\left(Z_{4}-I_{d}\right) / T_{d}, \\
\Delta I & =0, \text { for } 0 \leqslant t \leqslant t_{1}, \\
& =\Delta I_{\max }\left(t-t_{1}\right) /\left(t_{2}-t_{1}\right), \text { for } t_{1} \leqslant t \leqslant t_{2}, \\
& =\Delta I_{\max }, \text { for } t>t_{2} .
\end{aligned}
$$

The expressions for $Z_{3}$ and $Z_{4}$ are the same as given earlier.

It is assumed that the disturbance originates at $t=0^{+}$and the time required to sense this is $t_{1}$. In this type of controller, additional capacitors are switched on at time $t_{2}$ to meet the increased reactive power requirements at the converter buses due to increased current order.

\subsection{Case study and results}

The disturbance considered is a 3-phase-fault at $t=0$ at bus 5 followed by clearing of the fault by switching lines 5-7 off. For simplicity, the load characteristics are assumed to be of constant impedance type. The following cases are considered:

case 1 - with constant current reference (no modulation),

case 2 - with auxiliary controller $K_{w}=3$,

case 3 - with emergency controller.

In case 3 , it is assumed that an additional capacitor bank (of 0.25 p.u.) is added at both converter buses after time $t_{2}$ to account for the increased reactive power requirements.

The critical clearing time $\left(T_{c r}\right)$ obtained by digital simulation is $0 \cdot 164-0.165 \mathrm{~s}$ for case $1,0 \cdot 161-0 \cdot 162 \mathrm{~s}$ for case 2 and $0 \cdot 173-0 \cdot 174 \mathrm{~s}$ for case 3 . The corresponding value of $T_{\text {cr }}$ obtained by prediction (using the PEBS method) is $0.159-0 \cdot 160,0 \cdot 158-0.159$ 

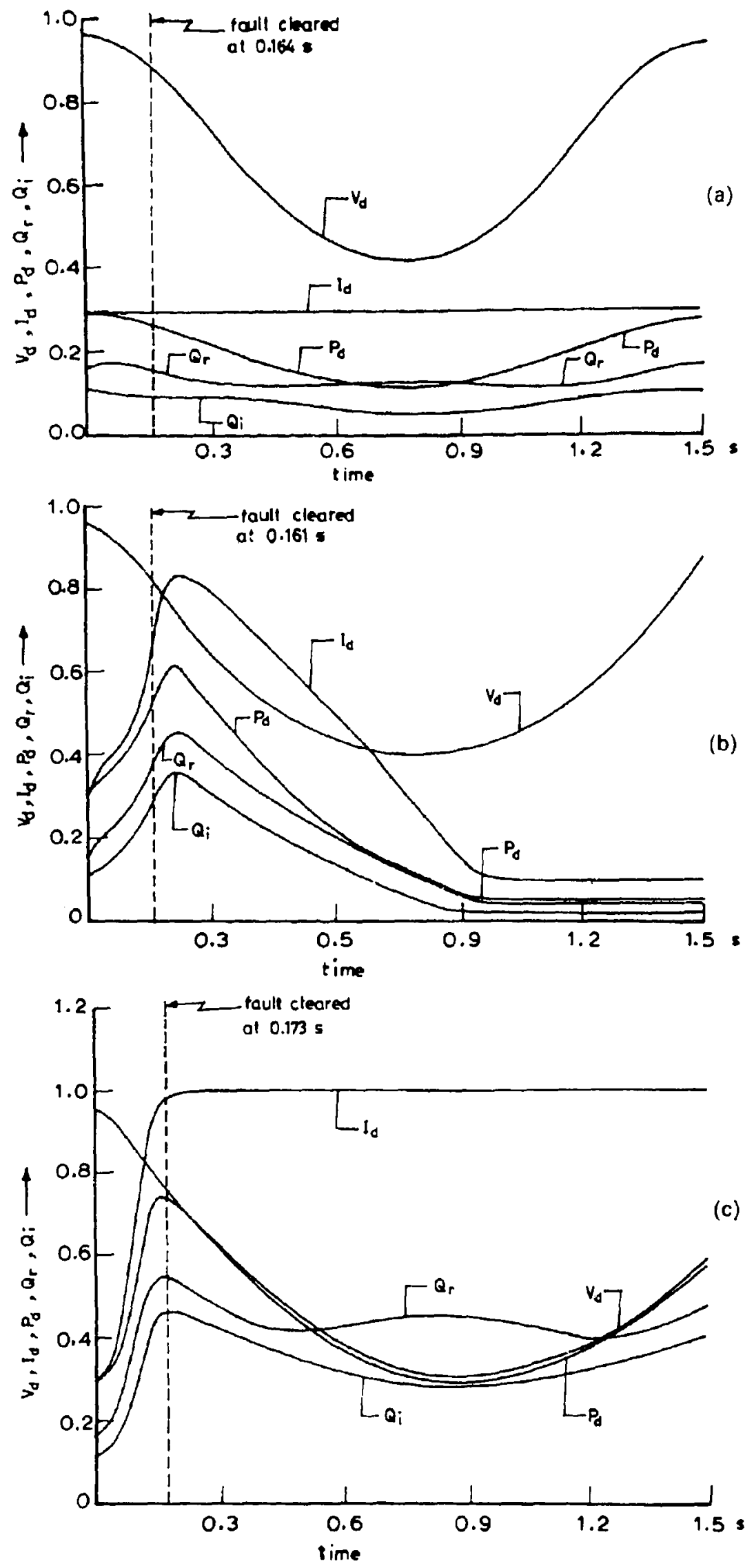

Figure 7. Variation of DC quantities. (a) Case 1 (stable); (b) case 2 (stable); (c) case 3 (stable). 
and $0.175-0.176 \mathrm{~s}$, respectively. The corresponding value of critical energy is 0.594 , 0.569 and 0.813 p.u., respectively. It is observed that $T_{c r}$ obtained by prediction agrees well with that obtained by digital simulation. $T_{c r}$ is slightly reduced for case 2 compared to that for case 1 . It is to be noted that the auxiliary controller is provided primarily for damping oscillations in the system. The effectiveness of this controller also depends on the choice of control parameters and the reactive power constraints. The latter can be explained as follows: As DC current is increased, the reactive power requirements are also increased. If adequate reactive power is not available, the AC voltage will drop, thus (partially) nullifying the effect of the controller.

As expected, the emergency controller helps in improving the transient stability. The results obtained by TEF are accurate enough to predict the effect of controllers. Figures $7 \mathrm{a}-\mathrm{c}$ show, for the post-fault system, the DC voltage $V_{\mathrm{d}}\left(=V_{d r}=V_{d i}\right.$ since $R$ is assumed to be zero), the DC link current $I_{\mathrm{d}}$, the reactive power $Q_{d r}, Q_{\mathrm{d} i}$ and the active DC power $P_{d}\left(=P_{d r}=P_{d i}\right)$, for cases 1-3, respectively, when the fault is assumed to be cleared critically. It is observed in the cases considered that $V_{d}$ decreases initially and then increases. The variation is greater in case 3 as compared to that in the other two cases. This is due to the variation in the AC bus voltages. The DC link current $I_{d}$ varies as expected in cases 2 and 3 ; however, its magnitude in case 2 is controlled by the AC bus frequency signal. In this case, it is observed that the current increases initially and, sometime after the fault clearance, falls till it is limited to a minimum value by the limiter.

$D C$ power increase is not so significant because of reduction of $D C$ voltage even when the DC current is increased in case 3. Yet, when compared to cases 1 and 2, the transient stability is improved. In case 2, even though the current is initially increased, it decreases due to the control signal becoming negative, and the current is prevented from going below the minimum value by the limiter. This shows that the auxiliary controller considered here has not helped in improving the transient stability.

Figures $8 \mathrm{a}$ and $\mathrm{b}$ show the variation of the total, potential and kinetic energies for case 3 for (a) stable, and (b) urstable conditions. In general, the total energy increases from its initial value $(=0)$ till the fault is cleared and remains constant afterwards, as expected. However, a slight increase in the total energy can be observed some time after the fault clearance. This may be due to the numerical errors (truncation and round-off) introduced in the evaluation of the energy function. In the cases depicted, it can be observed that the potential energy is initially negative and then increases. This is because the post-fault configuration considered is different from the prefault one because of line switching. As regards the kinetic energy, it can be observed that when the system is stable, this energy increases until the fault is cleared and then reduces to zero after some time. This is expected for the first swing stability.

\section{Conclusions}

A structure-preserving energy function is proposed for $A C / D C$ systems, which is general enough to cover two or multiterminal DC links, simplified or detailed converter controller models. As a first approximation, the energy function can be formulated as path-independent. 
(a)

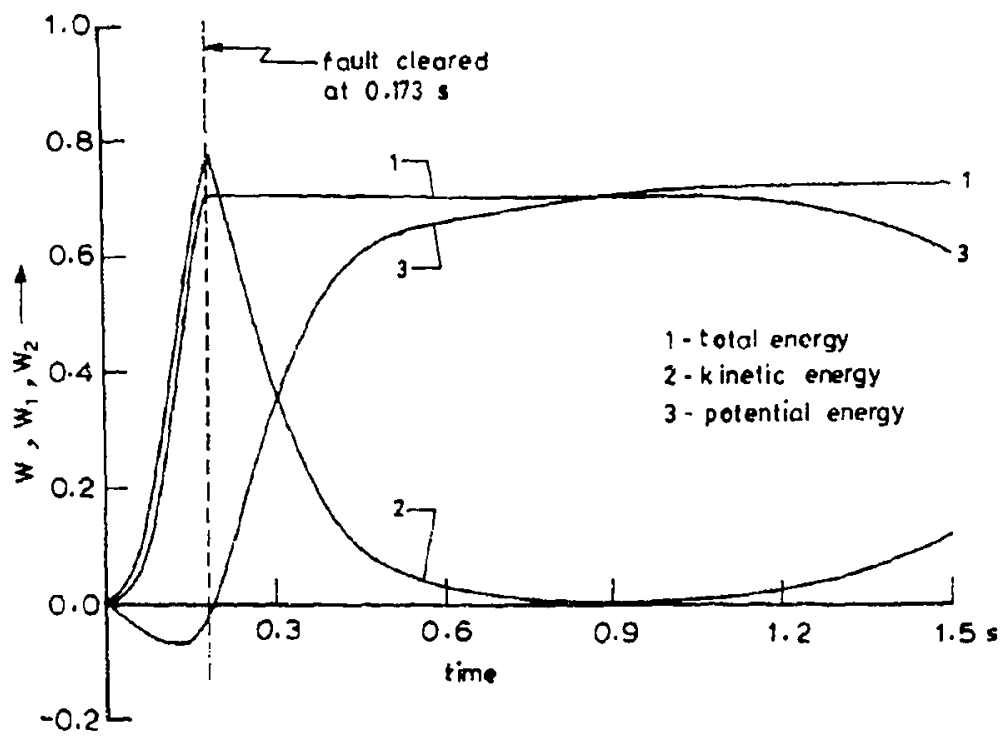

(b)

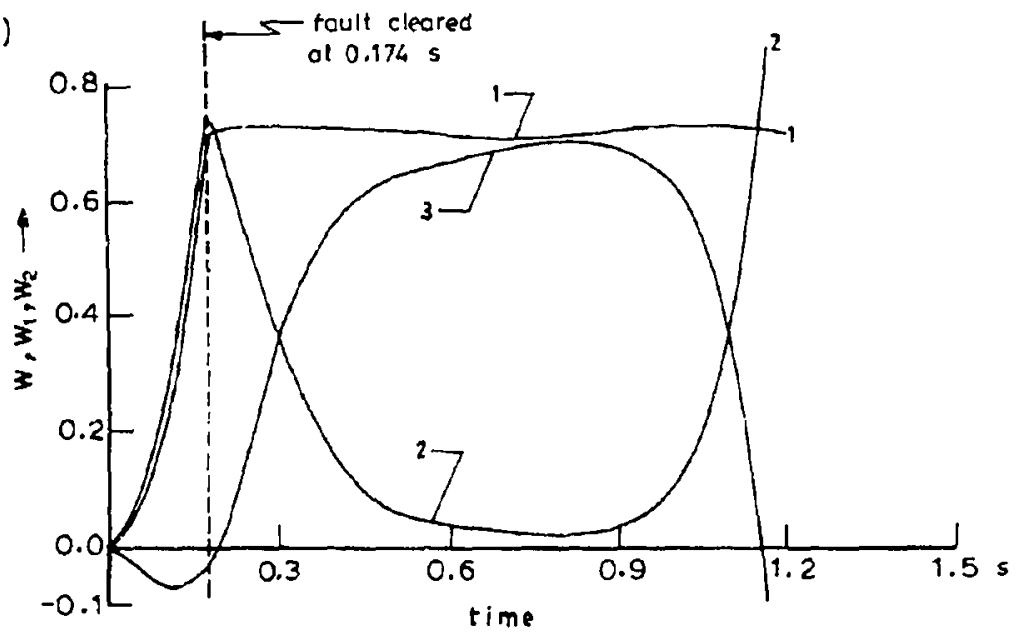

Figure 8. Variation of total, kinetic and potential energies. Case 3 - stable (a) and unstable (b).

Stability analysis carried out on a 3-machine system indicates the following.

(1) A DC link can improve the transient stability of the overall system. However, proper controllers have to be used to exploit this advantage of DC links;

(2) $T_{c r}$ predicted by the direct method using SPEF gives accurate results.

\section{References}

Anderson P M, Fouad A A 1977 Power system control and stability (Iowa: StateUniversity Press) Bergen A R, Hill D J 1981 A structure preserving model for power system stability analysis. IEEE Trans. Power Appar. Syst. PAS-100: 25-35

DeMarco C L, Canizares C A 1992 A vector energy function approach for security analysis of AC/DC systems. IEEE Trans. Power Syst. PWRS-7: 1000-1011

Fudeh H, Ong C M 1981 A simple and efficient AC-DC load flow method for multiterminal DC systems. IEEE Trans. Power Appar. Syst. PAS-100: 4389-4396

Hiskens I A, Hill D J 1989 Energy functions, transient stability and voltage behaviour in power systems with nonlinear loads. IEEE Trans. Power. Syst. PWRS-4: 1525-1533 
Lefebvre S, Wang W K, Reeve J, Gagnon J, Johnson B K 1991 Experience with modelling MTDC systems in transient stability programs. IEEE Trans. Power Delivery PWRD-6: 405-413

Narasimhamurthi N, Musavi M T 1984 A general energy function for transient stability analysis of power systems. IEEE Trans. Circuits. Syst. CAS-31: 637-645

Ni Y-X, Fouad A A 1987 A simplified two-terminal HVDC model and its use in direct transient stability assessment. IEEE Trans. Power Syst. PWRS-2: 1006-1012

Padiyar K R 1991 HVDC power transmission systems - Technology and system interactions (New Delhi: Wiley Eastern)

Padiyar K R, Ghosh K K 1987 A novel structure preserving energy function for dynamic stability evaluation of power systems with known modes of instability. Elec. Mach. Power Syst. 13: 135-148

Padiyar K R, Ghosh K K 1989 Direct stability evaluation of power systems with detailed generator models using structure-preserving energy functions. Int. J. Elec. Power Energy Syst. 11: 47-56

Padiyar K R, Sastry H S Y 1983 Application of topological energy function for the direct stability evaluation of power systems with voltage dependent loads. Proc. Int. Conf. on Systems, Man and Cybernetics (New York: IEEE Press)

Padiyar K R, Sastry H S Y 1984 Direct stability analysis of AC/DC power systems using a structure preserving energy function. Proc. Int. Conf. Comput. Syst. Signal Process. Bangalore, India

Padiyar K R, Sastry H S Y 1987 Topological energy-function analysis of stability of puwer systems. Int. J. Elec. Power Energy Syst. 9: 9-16

Pai M A 1989 Energy function analysis for power system stability (Boston: Kluwer)

Pai M A, Padiyar K R, Radhakrishna C 1981 Transient stability of multimachine AC/DC power systems via energy function method. IEEE Trans. Power Appar Syst. PAS-100: 5027-5035

Ribbens-Pavella M, Evans F $\ 1985$ Direct methods for studying dynamics of large-scale electric power systems - A survey. Auromatica 21: 1-21

Tsolas N, Arapostathis A, Varaiya P 1985 A structure-preserving energy function for power system transient stability analysis. IEEE Trans. Circuits Syst. CAS-32: 1041-1049

Varaiya P P, Chen R L, Wu F F 1985 Direct methods for transient stability analysis of power systems - Recent results. Proc. IEEE 73: 1703-1715 